\title{
Efectos ambientales sobre la variabilidad espacial del ictioplancton de Chile austral durante noviembre de 2005
}

\author{
Environmental effects on the spatial variability of the ichthyoplankton from southern \\ Chile during November 2005
}

CLAUDIA A. BUSTOS ${ }^{1, *}$, MAURICIO F. LANDAETA ${ }^{1} \&$ FERNANDO BALBONTÍN $^{2}$

\author{
${ }^{1}$ CIEN Austral- Centro de Investigación en Nutrición, Tecnología de Alimentos y Sustentabilidad, Universidad Austral de \\ Chile, Campus Puerto Montt, Chile \\ ${ }^{2}$ Facultad de Ciencias del Mar y de Recursos Naturales, Universidad de Valparaíso, Chile \\ *e-mail para correspondencia: claudiabustos@uach.cl
}

\begin{abstract}
RESUMEN
Se analiza la distribución y abundancia del ictioplancton de Chile austral $\left(41-45^{\circ} \mathrm{S}\right)$ durante noviembre de 2005 en relación a las características oceanográficas de la columna de agua (temperatura, salinidad y estratificación). Durante el periodo de estudio, se recolectó un total de 44.606 huevos de peces y 2.016 larvas correspondientes a 28 taxa. Los estados tempranos de peces pelágicos pequeños dominaron en las muestras, con densidades de $\sim 14.000$ huevos $10 \mathrm{~m}^{-2}$ de anchoveta (Engraulis ringens) y $>100$ larvas $10 \mathrm{~m}^{-2} \mathrm{de}$ clupeidos. Análisis multivariados indicaron la formación de dos grupos de estaciones, uno asociado a la zona de fiordos, de mayor profundidad, con fuertes gradientes verticales de temperatura y salinidad y mayor estratificación, y otro grupo asociado al borde oriental de la isla Chiloé, de menor profundidad y mayor mezcla en la columna de agua. En las zonas de mayor estratificación (i.e., mayor frecuencia de Brunt-Väisäla, $\mathrm{N}^{2}$ ), se encontraron mayores densidades de huevos y larvas de peces pelágicos y mesopelágicos (Strangomera bentincki y Maurolicus parvipinnis). Al comparar esta información con un crucero oceanográfico llevado a cabo 10 años antes en la misma zona (octubre de 1995), se detectaron cambios en la composición específica de ictioplancton, lo que puede indicar diferencias en las especies de peces marinos que utilizan la zona de fiordos y canales como área de desove y crianza larval.
\end{abstract}

Palabras clave: mar interior, fiordos, larvas de peces, frecuencia de Brunt-Väisäla, factores ambientales.

\begin{abstract}
The distribution and abundance of ichthyoplankton from southern Chile $\left(41-45^{\circ} \mathrm{S}\right)$ during November 2005 were analyzed, in relation to the oceanographic features of the water column (temperature, salinity, stratification). During the sampling period, 44.606 fish eggs and 2.016 fish larvae were collected, belonging to 28 taxa. Early life stages of small pelagic fishes dominated the plankton samples, with mean densities of 14,000 eggs $10 \mathrm{~m}^{-2}$ of anchovy (Engraulis ringens) and $>100$ larvae $10 \mathrm{~m}^{-2}$ of clupleids. Multivariate analysis showed the conformation of two groups of stations, one associated to deeper fjord areas, with strong vertical gradients of temperature and salinity, and higher stratification. The other group was associated to the shallow and well-mixed eastern border of Chiloé Island. In areas with strong stratification (i.e., higher Brunt-Väisäla frequency, $\mathrm{N}^{2}$ ), significant higher densities of pelagic and mesopelagic fish eggs and larvae were collected (i.e., Strangomera bentincki, Maurolicus parvipinnis). Comparing these results with an oceanographic cruise carried out 10 years before in the same area (October 1995), changes in the ichthyoplankton composition were detected; this may be caused by potential differences in the marine fish species which utilize fjords and channels of southern Chile as spawning and early nursery zone.
\end{abstract}

Key words: inland sea, fjords, fish larvae, Brunt-Väisäla frequency, environmental factors.

\section{INTRODUCCIÓN}

La variabilidad en la distribución espaciotemporal de los estados tempranos de especies marinas es producida por fluctuaciones de diversas características hidrodinámicas del sistema, y su interacción con patrones reproductivos, adaptaciones conductuales y diferencias en la sobrevivencia de los diferentes estados de desarrollo (Palma \& Silva 2004, 
Landaeta et al. 2006, Yannicelli et al. 2006). En sistemas de fiordos, varios procesos oceanográficos pueden influir en la composición y abundancia del zoo e ictioplancton, entre ellos la circulación estuarina (Palma \& Silva 2004, Landaeta \& Castro 2006), corrientes costeras (Lanksbury et al. 2005), mareas (Jager 2001) y la estratificación de la columna de agua (Lee et al. 2005, Bustos et al. 2007). A su vez, los organismos planctónicos presentan conductas de migración vertical diaria y/o migraciones ontogenéticas que les permiten mantenerse y/o ser transportados hacia áreas favorables para su crecimiento y sobrevivencia (Lopes 1979, Bailey \& Picquelle 2002, Landaeta \& Castro 2002).

En la zona austral de Chile muchas especies de peces, algunas de interés comercial (merluza del sur, Merluccius australis, merluza de cola Macruronus magellanicus, congrio Genypterus blacodes), presentan parte de su ciclo de vida en la zona dominada por la dinámica de fiordos (Balbontín \& Bravo 1993, Balbontín \& Bernal 1997, Landaeta \& Castro 2006, Bustos et al. 2007). Sin embargo, aun existe poca información sobre cómo interactúan las variables físicas y los procesos oceanográficos en la dinámica de los estados tempranos de peces marinos en el mar interior de Chile austral.

El objetivo del presente trabajo es determinar la heterogeneidad espacial en la composición y abundancia del ictioplancton en el sistema de fiordos del sur de Chile, en relación a las condiciones hidrográficas (temperatura, salinidad, estratificación de la columna de agua) presentes durante noviembre de 2005, y establecer explicaciones potenciales de los mecanismos físicos que modulan la dinámica de los estados tempranos de peces en ese ecosistema.

\section{MATERIALES Y MÉTODOS}

\section{Área de estudio}

El sistema de fiordos y canales de Chile austral es una zona conectada con el océano abierto por el norte a través del canal Chacao y por el sur por la boca del Guafo (Fig. 1). Esta área está dominada por vientos sur y suroeste durante primavera y verano (octubre-marzo) y vientos norte y noroeste durante otoño e invierno (abril-septiembre). Las mareas son semidiurnas, presentando variaciones de hasta 6 m (Cáceres et al. 2003), las que generan corrientes mareales medias típicas de 25-30 $\mathrm{cms}^{-1}$ en aguas superficiales (Salinas \& Castillo $^{1}$ ). El ingreso de agua dulce al sistema proviene de ríos que drenan desde los Andes y la alta pluviosidad, y que generan una marcada haloclina somera $(\sim 20 \mathrm{~m}$ de profundidad $)$ y una circulación tipo estuarina (Silva et al. 1998).

\section{Trabajo en terreno}

Se recolectaron muestras de zooplancton durante el crucero CIMAR 11 FIORDOS etapa 2 , llevado a cabo entre el 11 y 21 de noviembre de 2005 en la zona del mar interior de Chiloé, X Región de Chile (Fig. 1) a bordo del AGOR Vidal Gormaz. Las estaciones de muestreo estuvieron ubicadas en fiordos continentales (estuario Reloncaví, fiordos Comau y Hornopirén), en el borde oriental de la isla Chiloé y a lo largo de una transecta entre el estuario Reloncaví y la boca del Guafo. En un total de 72 estaciones se realizaron perfiles verticales de temperatura, salinidad, densidad y oxígeno disuelto utilizando un CTD Seabird SBE-25 desde la superficie hasta $500 \mathrm{~m}$ de profundidad o cerca del fondo.

El ictioplancton fue recolectado utilizando una red Bongo estándar $(60 \mathrm{~cm}$ de diámetro, redes de $300 \mu \mathrm{m}$ de apertura de malla) con flujómetros OSK para estimar el volumen filtrado en un total de 37 estaciones, donde se realizaron lances oblicuos hasta una profundidad máxima de $200 \mathrm{~m}$ o a $10 \mathrm{~m}$ cerca del fondo en aguas más someras, a una velocidad de 1-2 nudos. El volumen filtrado en cada lance (rango $=23-209 \mathrm{~m}^{3}$ por lance; media $=96 \mathrm{~m}^{3}$ por lance) fue estimado tomando en consideración el área de la boca y la lectura del flujómetro. Después de la finalización de cada muestreo, las redes fueron lavadas y las muestras fijadas y preservadas una en formalina al 5\% tamponeada con borato de sodio y otra en etanol al $90 \%$.

\footnotetext{
Salinas S \& M Castillo (2005) Corrientes de marea en el Paso Desertores $\left(42^{\circ} 45^{\prime} \mathrm{S}, 72^{\circ} 45^{\prime} \mathrm{W}\right)$, durante el período de invierno-primavera de 2004. Informes Preliminares Crucero Cimar 10 Fiordos, pp. 27-31.
} 


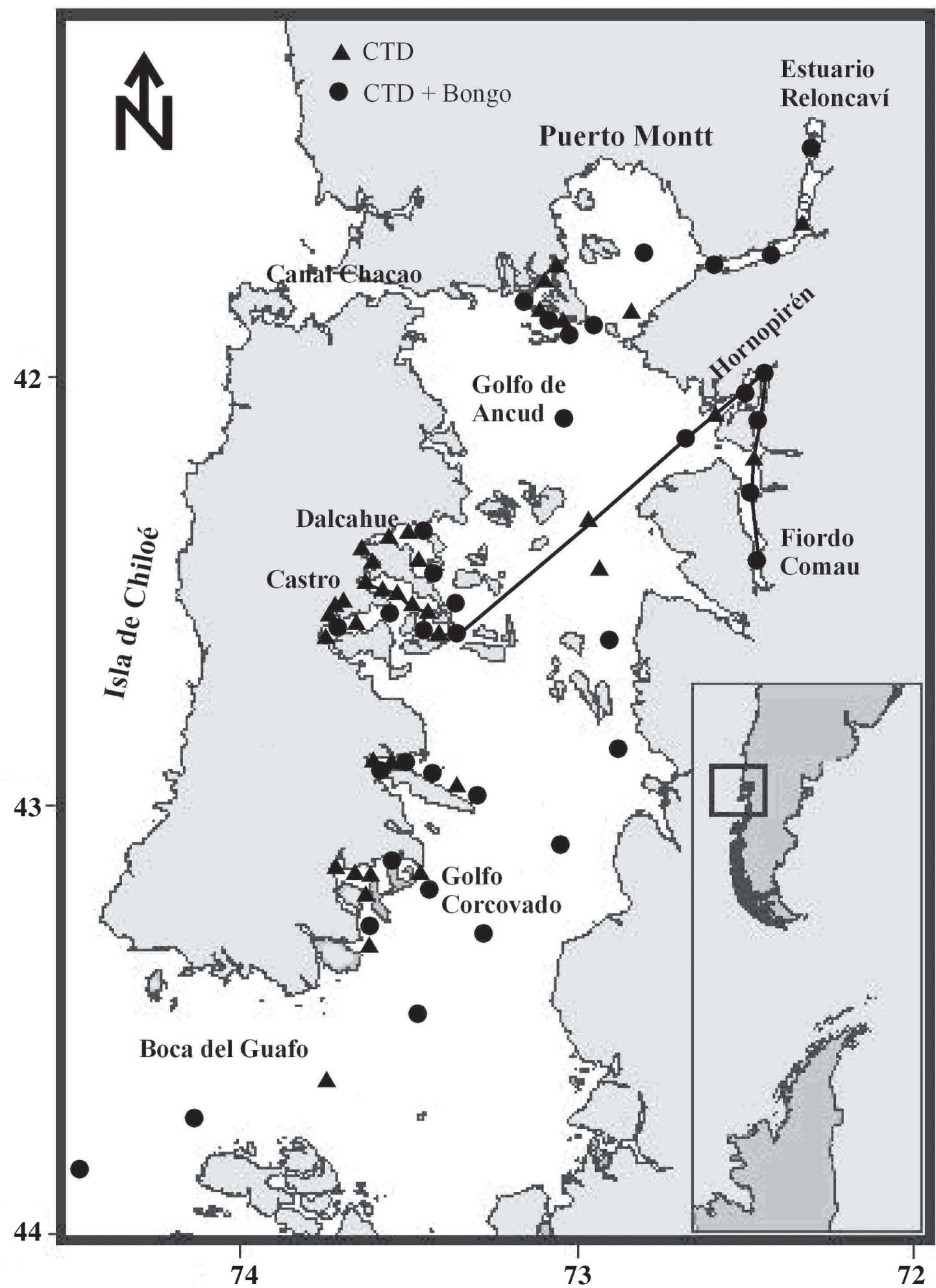

Fig. 1: Mapa del área de estudio indicando las estaciones oceanográficas (CTD, $\mathbf{\Delta}$ ) y de plancton muestreadas (CTD + Bongo, O) durante noviembre de 2005. La línea negra indica la sección longitudinal mostrada en la Fig. 5.

Map of the study area showing the sampled oceanographic (CTD, $\mathbf{\Delta})$ and plankton stations $(\mathrm{CTD}+\mathrm{Bongo}, \mathbf{0})$ during November 2005. Black line indicates the longitudinal section showed in Fig. 5. 
Los huevos y larvas fueron identificados en el laboratorio hasta el nivel taxonómico más bajo posible usando las descripciones de Orellana \& Balbontín (1983), Moser (1996), Neira et al. (1998), Balbontín et al. (2004), Bustos \& Landaeta (2005) y Uribe \& Balbontín (2005). En el uso de la nomenclatura para los nombres científicos de los peces se siguió a Eschmeyer (2007). Las larvas fueron contadas de acuerdo a su desarrollo ontogenético como en preflexión y postflexión notocordal. La densidad numérica de cada especie fue expresada como número de especímenes por 10 $\mathrm{m}^{2}$ de superficie de mar.

Adicionalmente, se calculó la frecuencia de Brunt-Väisäla $\left(\mathrm{N}^{2}\right)$ como indicador de la variabilidad en la estratificación de la columna de agua, según la ecuación: $\mathrm{N}^{2}=(\mathrm{g} / \rho) *(\partial \rho /$ $\partial \mathrm{z})$, donde $\mathrm{g}=$ gravedad de la Tierra $\left(9,8 \mathrm{~m}^{2} / \mathrm{s}\right)$, $\rho=$ densidad del agua, $z=$ profundidad.

\section{Análisis multivariados}

Para determinar la relación existente entre los grupos de taxa de larvas de peces y las estaciones se llevaron a cabo análisis multivariados, dendrogramas y escalamiento no métrico multidimensional (MDS), que son de frecuente uso en estudios de ictioplancton (Harris \& Cyrus 2000, Landaeta \& Castro 2004). Se categorizaron las estaciones de acuerdo a su ubicación en el borde oriental de la isla Chiloé (C), en los fiordos (F), en el golfo de Ancud (GA) y en el golfo Corcovado (GC). Las abundancias de las larvas de peces (individuos $10 \mathrm{~m}^{-2}$ ) se transformaron con $\log (\mathrm{x}$ + 1) para aumentar la contribución de los taxa menos abundantes y se generó una matriz de disimilitud de Bray-Curtis. El MDS fue utilizado cuando el valor de "stress" fue $<0,2$ (Harris \& Cyrus 2000). Finalmente se llevaron a cabo pruebas estadísticas no paramétricas utilizando el paquete computacional Statistica 6.0 .

Comparación con ictioplancton de primavera de 1995

Durante octubre-noviembre de 1995 se llevó a cabo un crucero de investigación (proyecto CIMAR-FIORDOS 1), en el cual se muestrearon 89 estaciones oceanográficas entre Seno del Reloncaví (4130’ S) y Laguna San
Rafael (47 $\left.{ }^{\circ} \mathrm{S}\right)$, utilizando la misma metodología descrita para el crucero de noviembre de 2005. A diferencia de la información previamente publicada (Balbontín \& Bernal 1997), en el presente trabajo se presentan y comparan a través de análisis no paramétricos (prueba U de Mann-Whitney) las abundancias larvales solamente de la zona comprendida entre el Seno de Reloncaví y la boca del Guafo, y que corresponde a 34 estaciones oceanográficas.

\section{RESULTADOS}

Variabilidad espacial del ictioplancton en el mar interior durante noviembre de 2005

Se analizó un total de 37 muestras de zooplancton provenientes del sistema de fiordos de la zona austral de Chile. Se separó un número total de 44.606 huevos de peces y 2.016 larvas de peces correspondientes a 28 taxa (Tabla 1). Se detectó una alta incidencia de huevos de anchoveta Engraulis ringens, del pez mesopelágico Maurolicus parvipinnis y de clupeidos (Fig. 2, Tabla 1). Los huevos de clupeidos presentaron un amplio rango de tamaño $(0,88-1,19 \mathrm{~mm}$ de diámetro, media \pm desviación estándar $=1,06 \pm 0,08 \mathrm{~mm}, \mathrm{n}=$ 59); sin embargo, no se logró determinar si los huevos pertenecían a sardina común (Strangomera bentincki) o sardina fueguina (Sprattus fueguensis) pues ambos huevos presentan caracteres similares (Ciechomski 1972, Herrera et al. 1987). Los huevos de Clupeidae estuvieron localizados principalmente en la costa oriental de la isla Chiloé (> 500 huevos $10 \mathrm{~m}^{-2}$, Fig. 2) y en la cabeza del fiordo Hornopirén ( $>2.000$ huevos $10 \mathrm{~m}^{-2}$ ). Tanto los huevos de M. parvipinnis como los de E. ringens se recolectaron en mayor abundancia en los fiordos y en el golfo de Ancud, reduciendo significativamente su densidad en la zona costera cercana a la isla Chiloé (M. parvipinnis: prueba U de MannWhitney, $\mathrm{U}=9, \mathrm{P}<0,001 ;$ E. ringens: $\mathrm{U}=$ 25, $\mathrm{P}<0,001 ;$ Fig. 2). Otros huevos importantes por su abundancia en la muestras fueron catalogados como Pinguipedidae, similares en forma a la descripción de Fischer (1958) de huevos del blanquillo Prolatilus jugularis. 


\section{TABLA 1}

Abundancia media (individuos $10 \mathrm{~m}^{-2}$ ), desviación estándar (de) y valores mínimos y máximos del ictioplancton presente en el sistema de fiordos de la región austral de Chile durante noviembre de 2005

Mean abundante (individuals $10 \mathrm{~m}^{-2}$ ), standard deviation (SD) and maximum and minimum values of ichthyoplankton collected in the fjord zone of southern Chile during November 2005

\begin{tabular}{|c|c|c|c|c|}
\hline Huevos & Media & $\mathrm{DE}$ & Min & $\operatorname{Max}$ \\
\hline Engraulis ringens & $14.696,92$ & $47.780,24$ & 31,01 & $248.952,59$ \\
\hline Maurolicus parvipinnis & 794,01 & $1.348,65$ & 7,78 & $5.907,88$ \\
\hline Clupeidae & 491,61 & 615,77 & 4,30 & $2.339,23$ \\
\hline Pinguipedidae & 164,87 & 125,27 & 25,63 & 322,51 \\
\hline Merluccius gayi & 123,49 & 116,71 & 14,04 & 386,65 \\
\hline Stromateus stellatus & 120,12 & 19,45 & 106,37 & 133,87 \\
\hline Macrouridae & 36,59 & 48,49 & 7,17 & 133,54 \\
\hline Normanichthys crockeri & 34,26 & 28,18 & 4,44 & 110,08 \\
\hline Hippoglossina mystacium & 24,75 & 15,26 & 4,30 & 47,54 \\
\hline Genypterus sp. & 18,17 & 14,59 & 6,09 & 34,39 \\
\hline Congiopodus peruvianus & 14,74 & 4,04 & 11,89 & 17,60 \\
\hline Macruronus magellanicus & 13,31 & & 13,31 & 13,31 \\
\hline No Identificado & $2.263,49$ & $3.325,05$ & 6,09 & $13.514,66$ \\
\hline \multicolumn{5}{|l|}{ Larvas } \\
\hline \multicolumn{5}{|l|}{ Epipelágicos } \\
\hline Engraulis ringens & 368,45 & 707,69 & 4,30 & $2.640,07$ \\
\hline Normanichthys crockeri & 105,46 & 139,59 & 4,78 & 725,97 \\
\hline Strangomera bentincki & 63,26 & 56,25 & 4,44 & 209,28 \\
\hline Sprattus fueguensis & 38,70 & 20,76 & 17,60 & 59,09 \\
\hline Stromateus stellatus & 23,58 & 7,96 & 16,52 & 32,20 \\
\hline \multicolumn{5}{|l|}{ Mesopelágicos } \\
\hline Maurolicus parvipinnis & 195,68 & 189,40 & 6,09 & 657,02 \\
\hline Lampanyctodes hectoris & 21,51 & & 21,51 & 21,51 \\
\hline Bathylagichthys parini & 13,31 & & 13,31 & 13,31 \\
\hline \multicolumn{5}{|l|}{ Demersales-bentónicos } \\
\hline Merluccius gayi & 64,83 & 69,13 & 4,44 & 241,99 \\
\hline Merluccius australis & 31,12 & 25,70 & 13,14 & 70,30 \\
\hline Macrouridae & 27,08 & 17,80 & 6,68 & 39,42 \\
\hline Moridae & 22,26 & & 22,26 & 22,26 \\
\hline Paralichthys microps & 16,68 & 12,79 & 5,40 & 44,03 \\
\hline Hippoglossina mystacium & 12,87 & 9,98 & 4,78 & 32,20 \\
\hline Agonopsis chiloensis & 12,35 & 5,11 & 4,44 & 20,02 \\
\hline Macruronus magellanicus & 12,17 & & 12,17 & 12,17 \\
\hline Genypterus sp. & 9,53 & 3,34 & 7,17 & 11,89 \\
\hline \multicolumn{5}{|l|}{ Submareal-intermareal-estuarino } \\
\hline Sebastes capensis & 95,25 & 187,97 & 4,30 & 941,07 \\
\hline Pinguipes chilensis & 65,83 & 63,20 & 23,78 & 181,68 \\
\hline Hypsoblennius sordidus & 27,36 & 34,44 & 4,44 & 107,55 \\
\hline Galaxias maculatus & 17,19 & & 17,19 & 17,19 \\
\hline Leptonotus blainvilleanus & 15,58 & 11,19 & 6,09 & 35,15 \\
\hline Helcogrammoides cuninghami & 15,46 & 7,85 & 4,78 & 26,89 \\
\hline Prolatilus jugularis & 13,60 & 8,33 & 4,78 & 32,20 \\
\hline Odontesthes regia & 12,97 & 1,52 & 11,89 & 14,04 \\
\hline Helicolenus lengerichi & 7,17 & & 7,17 & 7,17 \\
\hline Gobiesox marmoratus & 6,09 & & 6,09 & 6,09 \\
\hline Ophiogobius jenynsi & 3,20 & & 3,20 & 3,20 \\
\hline No identificado & 30,89 & 41,95 & 4,44 & 194,03 \\
\hline
\end{tabular}



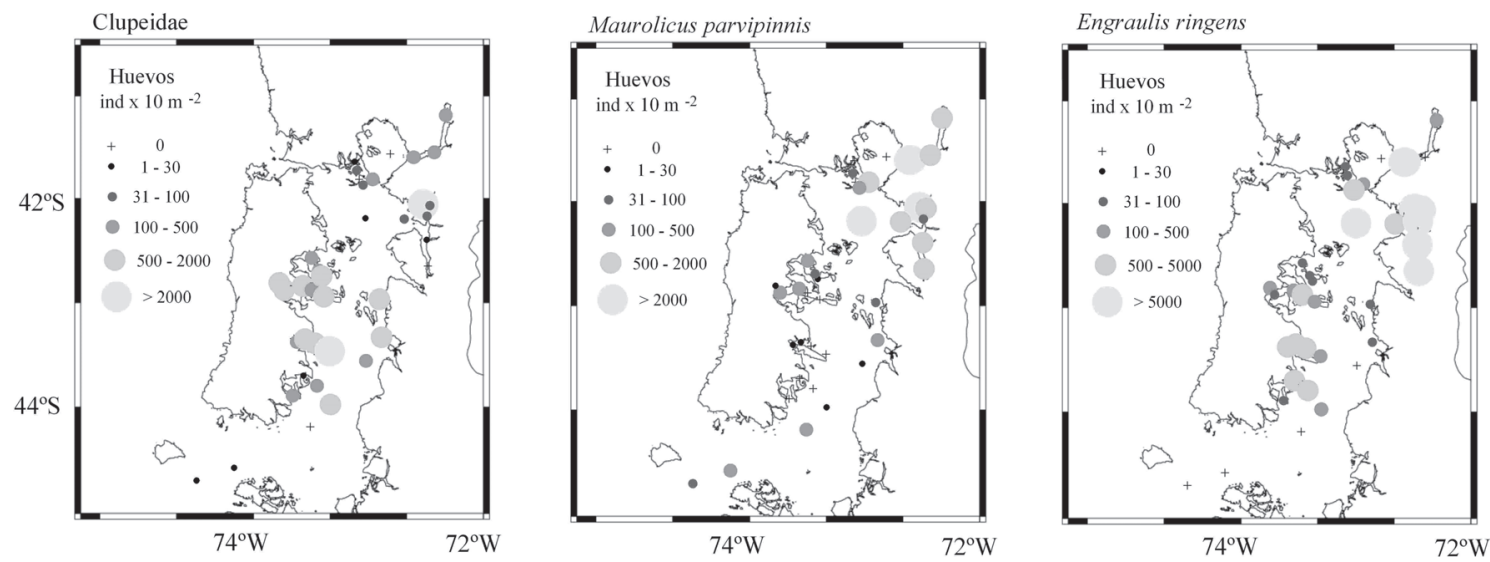

Fig. 2: Densidad de huevos de peces (individuos $10 \mathrm{~m}^{-2}$ ) recolectadas en las muestras de plancton en el sistema de fiordos de Chiloé, Chile austral.

Density of fish eggs (individuals $10 \mathrm{~m}^{-2}$ ) collected in plankton samples in the inland sea of southern Chile.

Con respecto a las larvas de peces, las larvas de peces epipelágicos dominaron en abundancia (principalmente larvas de anchoveta $E$. ringens y mote Normanichthys crockeri), seguidos por larvas mesopelágicas (M. parvipinnis), larvas de peces submareales (Sebastes capensis y Pinguipes chilensis) y finalmente larvas de adultos demersales (Merluccius gayi, $M$. australis) (Tabla 1). La distribución horizontal de las larvas de peces fue similar al patrón detectado en los huevos (Fig. 3). Las mayores abundancias ( $>100$ larvas $10 \mathrm{~m}^{-2}$ ) fueron encontradas en las zonas profundas del estuario Reloncaví y fiordos Hornopirén y Comau para estados tempranos de $S$. bentincki, $M$. parvipinnis y $S$. capensis. A diferencia de otras taxa, las larvas de mote $N$. crockeri se detectaron también en alta abundancia (entre 100 y 500 larvas $10 \mathrm{~m}^{-2}$ ) en las cercanías del estuario Castro y canal Dalcahue (Fig. 3). Sin embargo, solo se detectaron diferencias significativas entre el sector de fiordos y la zona correspondiente a la costa oriental de la isla Chiloé, para larvas de merluza común (prueba U de Mann-Whitney, $\mathrm{P}=0,011$ ).

Los análisis de clasificación y ordenación de las densidades larvales separaron dos grupos principales, a un nivel de similitud del $50 \%$ en el análisis de dendrogramas (Fig. 4A y 4B). El Grupo C consistió en la mayoría de las estaciones muestreadas en el borde oriental de la isla Chiloé y algunas estaciones en el golfo Corcovado, y el Grupo F son todas las estaciones llevadas a cabo en fiordos
(Hornopirén, Comau y estuario Reloncaví) (nivel de stress $=0,19$ ). Las especies responsables en la diferenciación de ambos grupos fueron las larvas de $M$. parvipinnis, E. ringens y $M$. gayi (Tabla 2).

\section{Distribución del ictioplancton y condiciones oceanográficas}

La Fig. 5 muestra los gradientes de temperatura y salinidad en una transecta longitudinal y la abundancia de larvas de mote Normanichthtys crockeri, merluza común Merluccius gayi y huevos y larvas de Maurolicus parvipinnis durante noviembre de 2005. En una sección transversal entre la isla Chiloé y los fiordos Hornopirén y Comau (Fig. 5) se observaron notorias diferencias en la estructura de la columna de agua de ambas zonas. Frente a Chiloé la temperatura no excedió los $12^{\circ} \mathrm{C}$ y la salinidad fue homogénea en toda la columna de agua $(\sim 32)$. En esta zona se detectaron bajas abundancias de larvas de mote $(<160$ individuos $\left.10 \mathrm{~m}^{-2}\right)$. En cambio, en la zona de fiordos se observó una zona frontal horizontal, y en los primeros $50 \mathrm{~m}$ de profundidad la temperatura varió entre 11 y $14^{\circ} \mathrm{C}$, presentando además fuertes gradientes verticales de salinidad (entre < 15 a 32). Precisamente en esta área fue donde se encontraron altas abundancias de larvas de mote (715 ind. por $10 \mathrm{~m} 2$ ), larvas de merluza común (hasta 240 larvas $10 \mathrm{~m}^{-2}$ ) y huevos (> 3.700 huevos $10 \mathrm{~m}^{-2}$ ) y larvas (>280 larvas $\left.10 \mathrm{~m}^{-2}\right)$ de M. parvipinnis (Fig. 5). 


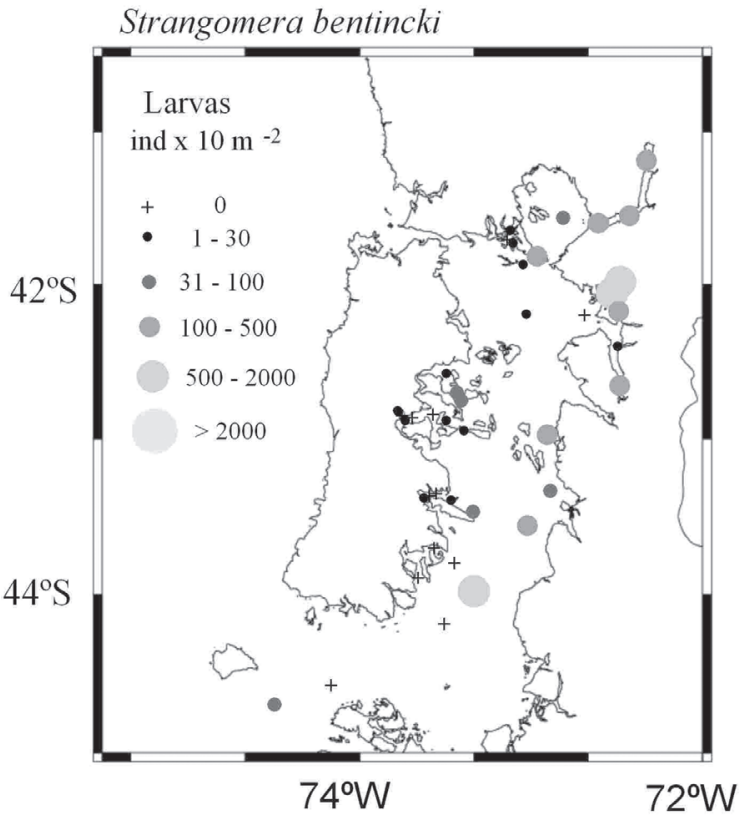

Normanichthys crockeri

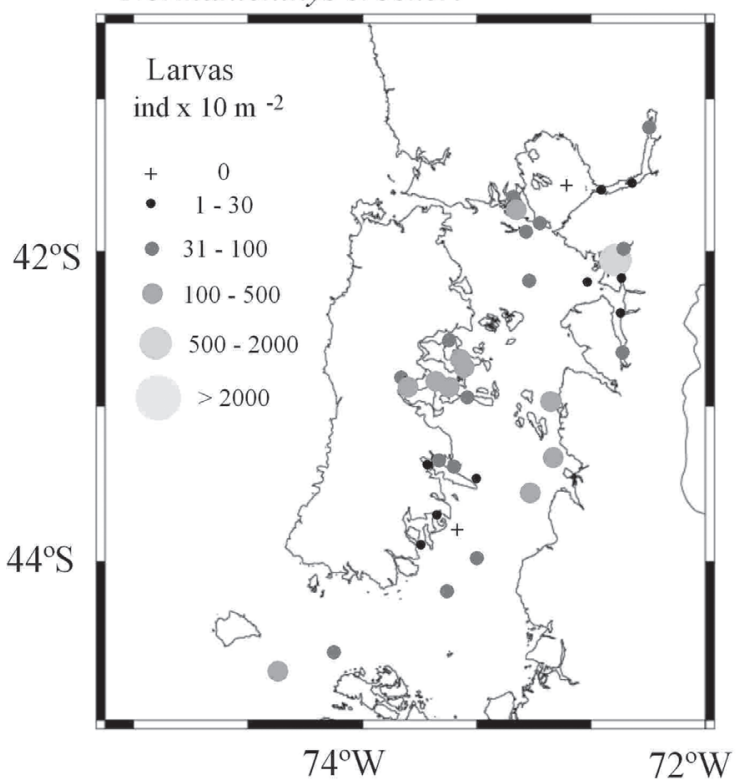

Maurolicus parvipinnis

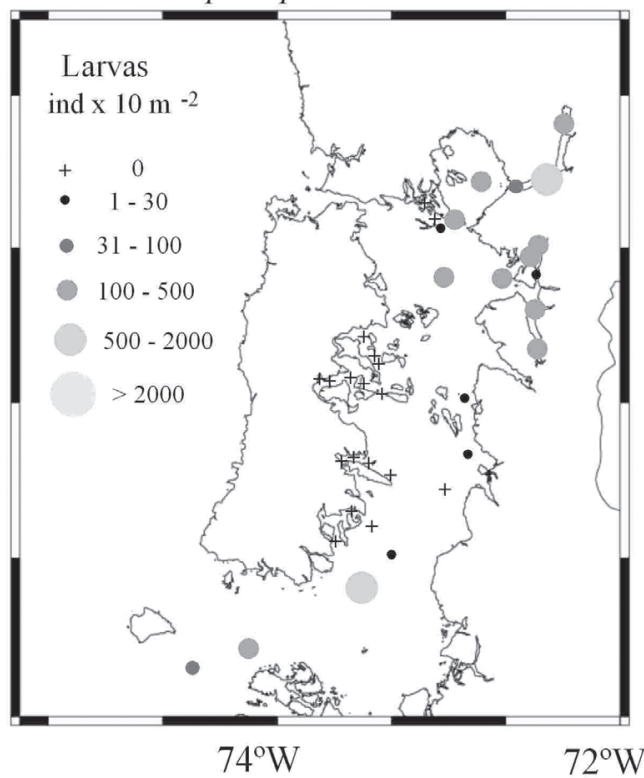

Sebastes capensis

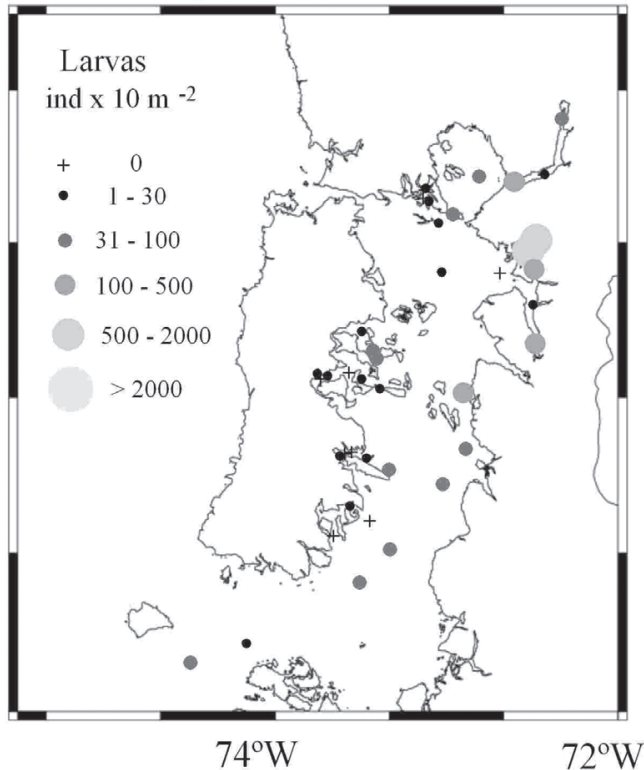

Fig. 3: Densidad de larvas de peces (individuos $10 \mathrm{~m}^{-2}$ ) recolectadas en las muestras de plancton en el mar interior de la X Región de Chile.

Density of fish larvae (individuals $10 \mathrm{~m}^{-2}$ ) collected in plankton samples in the inland sea of southern Chile.

Los mayores gradientes térmicos y salinos detectados en la zona de fiordos en comparación con lo observado en las cercanías de isla Chiloé y los golfos de Ancud y Corcovado generaron una columna de agua mucho más estratificada y estable (Fig. 6). La mayor frecuencia de Brunt-
Väisäla $\left(\mathrm{N}^{2}\right)$ se encontró asociada a la fuerte picnoclina (en los primeros $30 \mathrm{~m}$ de la columna de agua) y fue mayor en fiordos $\left(0,06 \mathrm{~s}^{-1}\right)$, en el golfo de Ancud $\left(0,01 \mathrm{~s}^{-1}\right)$, el golfo Corcovado $\left(0,0001 \mathrm{~s}^{-1}\right)$ y finalmente frente a Chiloé $(<2 \mathrm{x}$ $10^{-5} \mathrm{~s}^{-1}$, Fig. 6). 
(A)

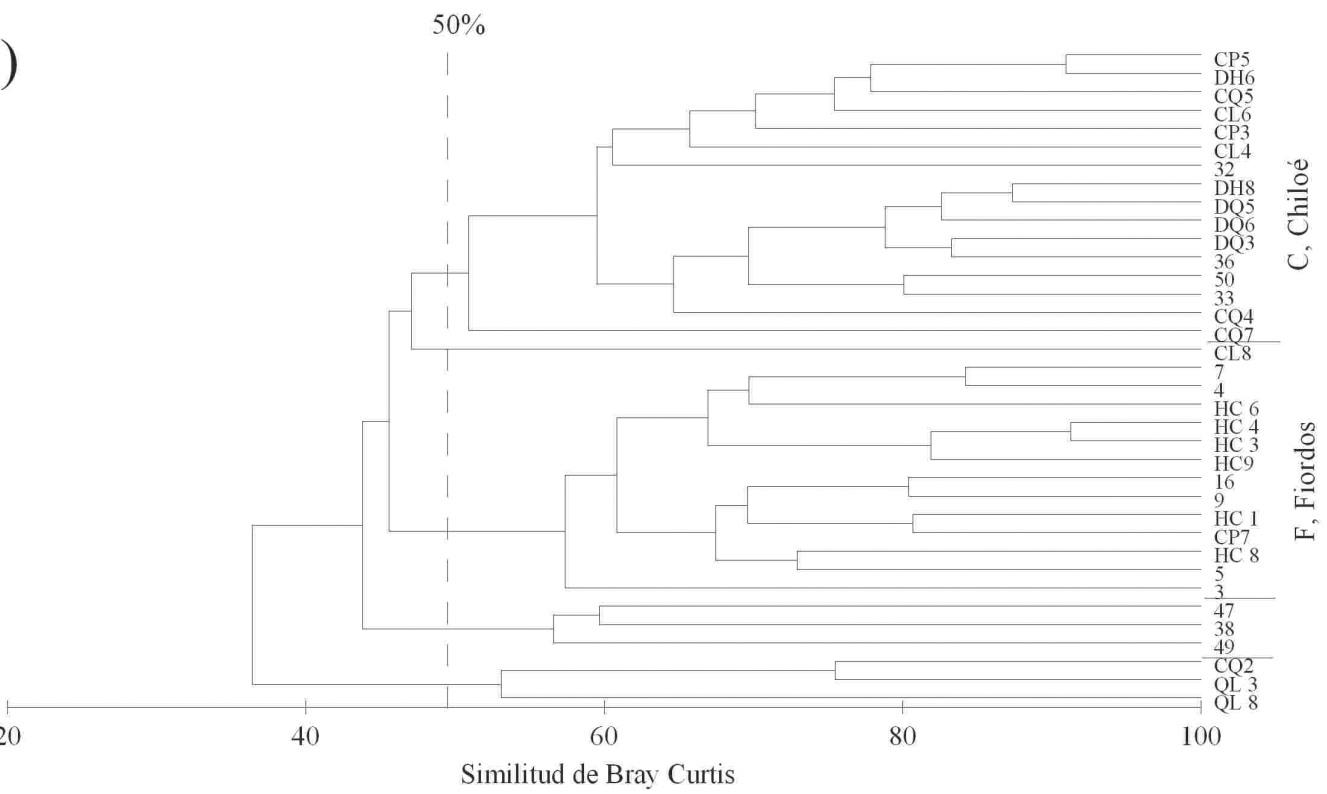

(B)

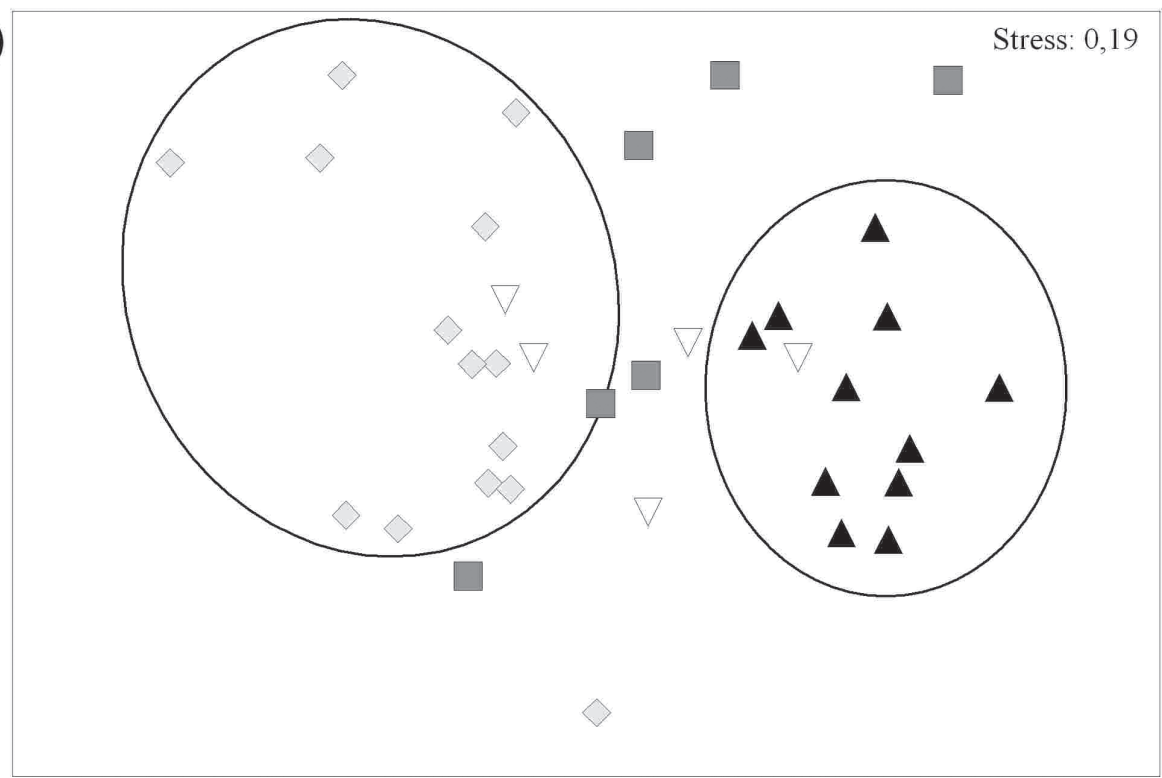

Fig. 4: Análisis multivariados sobre datos de abundancia de larvas de peces, (A) análisis de dendrogramas, (B) escalamiento no métrico multidimensional. Estaciones de muestreo ubicadas en $\mathrm{F}=$ fiordos, $\mathrm{GA}$ = Golfo de Ancud, $\mathrm{GC}=$ Golfo Corcovado, $\mathrm{C}=$ borde oriental de isla Chiloé.

Multivariate analysis on fish larvae abundance data, (A) cluster analysis, (B) non-metric multidimensional scaling. The sampling stations were located in fjords (F), Gulf of Ancud (GA), Gulf of Corcovado (GA) and off Chiloé Island (C). 
TABLA 2

Comparación del ictioplancton presente en la zona de fiordos (Grupo F) y en la costa oriental de la isla Chiloé (Grupo C) durante noviembre de 2005. DE = desviación estándar

Comparison of ichthyoplankton found in the fjord zone (Group F) and off the eastern coast of Chiloé Island (Group C) during November 2005. DE = standard deviation

\begin{tabular}{lccc}
\hline Especie & Grupo F abundancia media & Grupo C abundancia media & Disimilitud/DE \\
\hline Maurolicus parvipinnis & 207,08 & 0 & 2,72 \\
Engraulis ringens & 993,38 & 13,80 & 2,15 \\
Merluccius gayi & 69,35 & 0 & 1,89 \\
Sebastes capensis & 195,43 & 18,67 & 1,40 \\
Strangomera bentincki & 73,23 & 28,30 & 1,16 \\
Pinguipes chilensis & 35,91 & 0 & 1,04 \\
Normanichthys crockeri & 94,25 & 113,39 & 1,23 \\
Merluccius australis & 18,83 & 0 & 0,85 \\
Hypsoblennius sordidus & 17,46 & 2,88 & 0,87 \\
Paralichthys microps & 4,8 & 6,65 & 0,84 \\
Prolatilus jugularis & 3,36 & 4,84 & 0,84 \\
Helcogrammoides cunninghami & 5,24 & 1,57 & 0,78 \\
Stromateus stellatus & 5,85 & 1,57 & 0,65 \\
\hline
\end{tabular}

Comparación con ictioplancton de noviembre de 1995

Durante el crucero de primavera de 1995 se detectaron 16 taxa de larvas de peces en las muestras de zooplancton recolectadas en el mar interior de Chiloé (Tabla 3). Las larvas más abundantes correspondieron al pez luminoso $M$. parvipinnis, cabrilla $S$. capensis, merluza de cola Macruronus magellanicus y pejerrata (Macrouridae). Cuando fueron posibles, los análisis estadísticos no detectaron diferencias significativas $(\mathrm{P}<0,05$, Tabla 3$)$ a nivel específico entre los valores de abundancia larval entre 1995 y 2005 , a pesar de las diferencias en la riqueza específica (16 especies en 1995, 28 especies en 2005) y la frecuencia de ocurrencia de varias taxa larvales, como las larvas de pejerrata $(58,8$ versus $10,8 \%)$ y merluza de cola $(73,5$ versus $2,7 \%)$. Además, el ictioplancton de noviembre de 2005 mostró una mayor frecuencia de ocurrencia de larvas de peces epipelágicos como la anchoveta $(2,9$ $\%$ en 1995 y $81,1 \%$ en 2005, Tabla 3), sardina común y mote, estas dos últimas especies no presentes en el ictioplancton de primavera de 1995 (Tabla 3).

\section{DISCUSIÓN}

El análisis de los estados tempranos de peces presentes en las muestras de plancton durante noviembre de 2005 permitió identificar una alta variabilidad espacial tanto en composición como en abundancia del ictioplancton en el sistema de fiordos y canales de Chiloé. La zona de los fiordos Comau, Hornopirén y estuario Reloncaví, se caracteriza topográficamente por presentar bordes con fuerte pendiente y alta profundidad (> $200 \mathrm{~m}$ ), y oceanográficamente por una columna de agua con marcados gradientes térmicos y salinos, que generan una fuerte estratificación vertical (i.e., valores de frecuencia de Brunt-Väisäla de $0,06 \mathrm{~s}^{-1}$, Fig. 6). En estas áreas se detectaron los más altos números de taxa de huevos y larvas de peces de diferente origen adulto (epipelágico, demersal, mesopelágico y estuarino/submareal) y las mayores densidades de huevos de E. ringens y M. parvipinnis (Fig. 2 y 3), y larvas de sardina común Strangomera bentincki, cabrilla $S$. capensis, rollizo $P$. chilensis y merluza común M. gayi (Tabla 2). Por otro lado, tanto la abundancia como la composición específica del ictioplancton fue significativamente menor en 

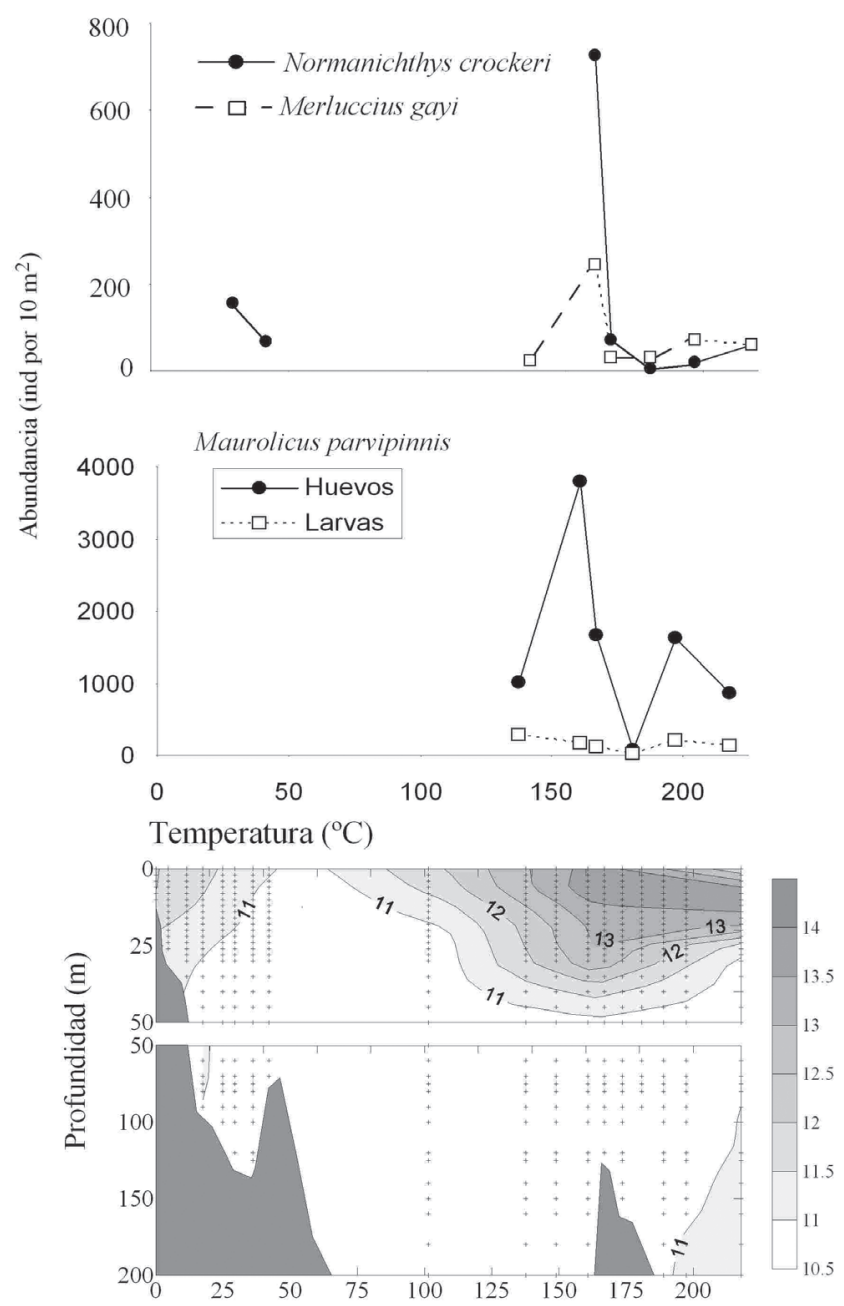

Salinidad

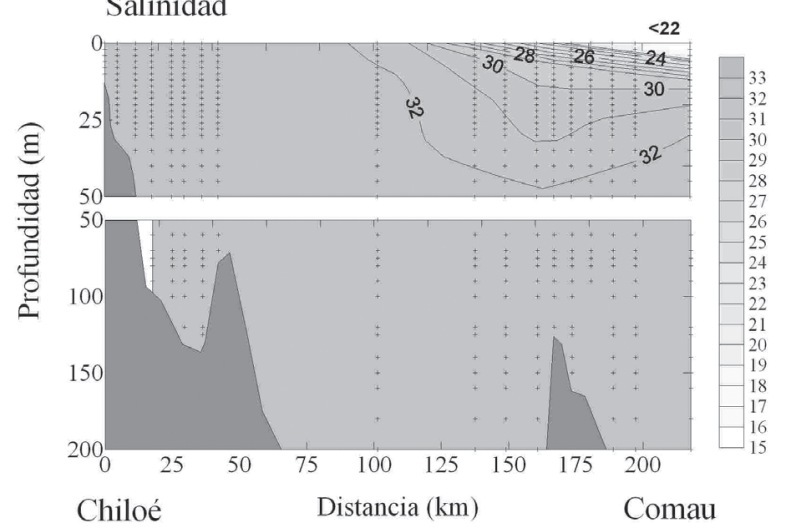

Fig. 5: Sección longitudinal de temperatura $\left({ }^{\circ} \mathrm{C}\right)$ y salinidad entre el borde oriental de la isla de Chiloé y fiordos Comau y Hornopirén, indicando la abundancia estandarizada de larvas de mote (Normanichthys crockeri) y merluza común (Merluccius gayi), y huevos y larvas de Maurolicus parvipinnis (individuos $10 \mathrm{~m}^{-2}$ ). Las cruces (+) indican los datos de CTD con los cuales se construyeron las secciones transversales.

Longitudinal section of temperature $\left({ }^{\circ} \mathrm{C}\right)$ and salinity between the oriental side of Chiloé Island and fjords Comau and Hornopirén, showing standardized abundance of larval mote sculpin (Normanichthys crockeri) and hake (Merlucius gayi), and eggs and larvae of Maurolicus parvipinnis (individuals $10 \mathrm{~m}^{-2}$ ). Crosses (+) indicate data from CTD utilized for the construction of transversal sections. 

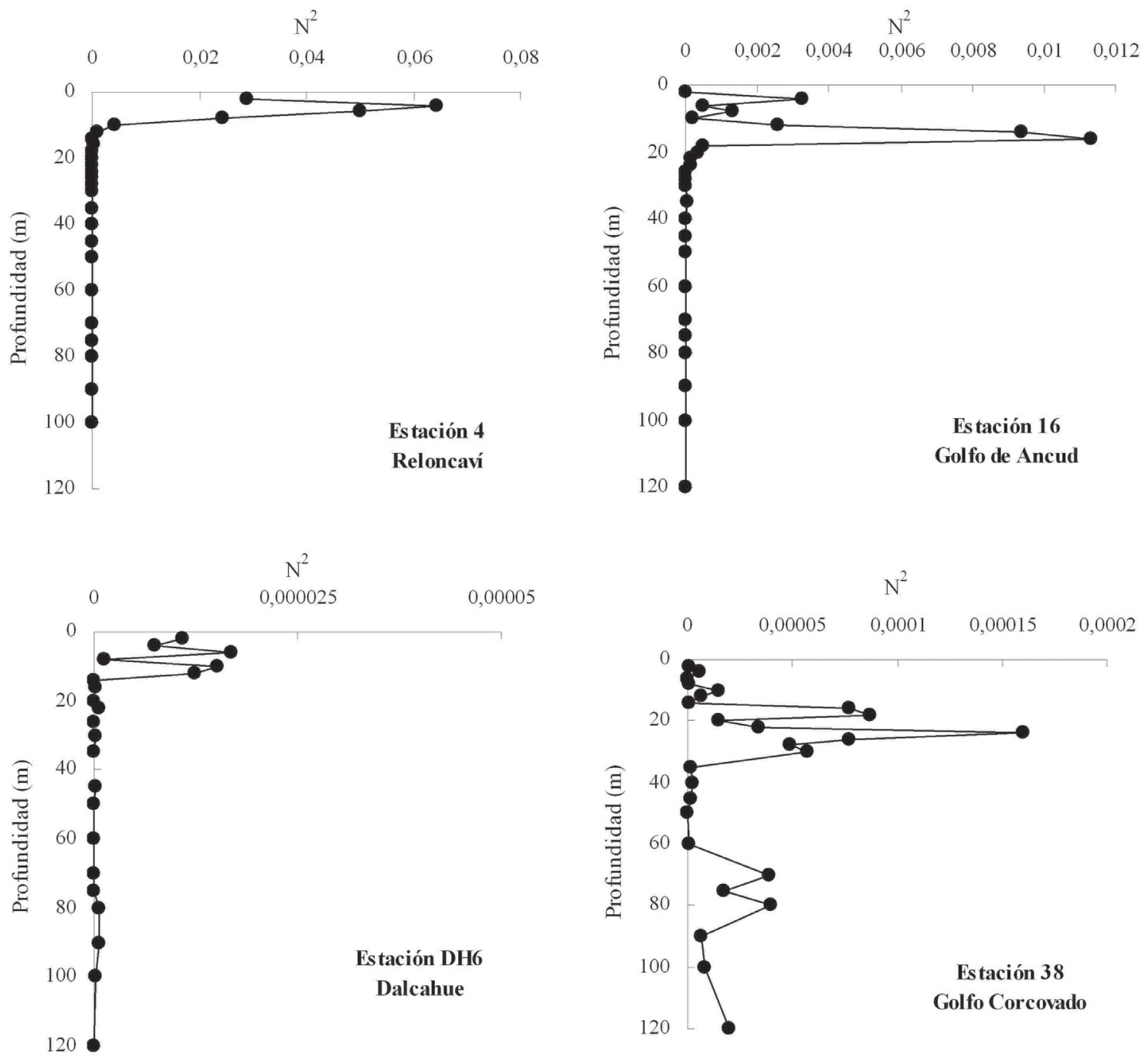

Fig. 6: Estratificación de la columna de agua, indicada por la frecuencia de Brunt-Väisäla $\left(\mathrm{N}^{2}\right)$ en cuatro estaciones del área de estudio.

Water column stratification, indicated by Brunt-Väisäla frequency $\left(\mathrm{N}^{2}\right)$ at four stations of the study area.

las aguas del sector oriental de la isla Chiloé. Esta zona se caracteriza por ser mucho más somera $(<100 \mathrm{~m})$ y tener menor aporte de agua dulce por ríos que el sector occidental de la cuenca interior. Estas características generan una mayor mezcla vertical y menor estratificación de la columna de agua (menores valores de $\mathrm{N}^{2}$ ) (Fig. 6).

Usando análisis de imágenes satelitales SeaWIFS se ha detectado en el mar interior de Chiloé una alta heterogeneidad espacial en la concentración de clorofila y producción primaria (Acha et al. 2004, Delgado \& Marín 2006, Iriarte et al. 2007). Los análisis muestran menores valores de estas dos variables en el borde oriental de la Isla de Chiloé, particularmente al sur de Castro y en el Golfo Corcovado $\left(<1,5 \mathrm{mg} \mathrm{m}^{-3} \mathrm{y}<2.000 \mathrm{mg} \mathrm{C} \mathrm{m}^{-2}\right.$ $\mathrm{d}^{-1}$, respectivamente) en comparación con las aguas del Golfo de Ancud (> $3 \mathrm{mg} \mathrm{m}^{-3} \mathrm{y}>$ $3.000 \mathrm{mg} \mathrm{C} \mathrm{m}^{-2} \mathrm{~d}^{-1}$, respectivamente) para el periodo de primavera (Iriarte et al. 2007). Similarmente, el zooplancton muestra también variabilidad espacial en su distribución; el sifonóforo Lensia conoidea presentó mayores concentraciones asociadas a aguas estuarinas de los fiordos de Chile austral durante primavera (Palma \& Silva 2004). 
TABLA 3

Mediana de la abundancia (individuos $10 \mathrm{~m}^{-2}$ ) de larvas de peces durante primavera de 1995, porcentaje de estaciones positivas y análisis estadístico de abundancia larval entre 1995 y 2005 en el mar interior de Chiloé

Median abundance (individuals $10 \mathrm{~m}^{-2}$ ) of fish larvae during austral spring 1995, percentage of positive stations and statistical analysis of larval abundance between 1995 and 2005 in the inner sea of Chiloé

\begin{tabular}{|c|c|c|c|c|c|}
\hline Taxa larval & Mediana & $\begin{array}{c}\text { Estaciones positivas } \\
\text { (\%) } 1995\end{array}$ & $\begin{array}{l}\text { Estaciones positivas } \\
\text { (\%) } 2005\end{array}$ & Valor de U & Valor de $\mathrm{P}$ \\
\hline Maurolicus parvipinnis & 110,3 & 58,8 & 51,3 & 187 & 0,930 \\
\hline Sebastes capensis & 87,2 & 70,6 & 86,5 & 271 & 0,061 \\
\hline Macrouridae & 35,5 & 58,8 & 10,8 & 27 & 0,313 \\
\hline Merluccius australis & 54,2 & 41,2 & 18,9 & 28 & 0,117 \\
\hline Macruronus magellanicus & 68,4 & 73,5 & 2,7 & - & - \\
\hline Seriolella sp. & 20,9 & 14,7 & - & - & - \\
\hline Congiopodus peruvianus & 8,6 & 8,82 & - & - & - \\
\hline Hypsoblennius sordidus & 17,6 & 2,9 & 27,0 & - & - \\
\hline Salilota australis & 13,3 & 2,9 & - & - & - \\
\hline Engraulis ringens & 37,8 & 2,9 & 81,1 & - & - \\
\hline Agonopsis chiloensis & 11,9 & 5,9 & 18,9 & - & - \\
\hline Hygophum brunni & 17,6 & 2,9 & - & - & - \\
\hline Lampanyctodes hectoris & 30,3 & 8,8 & 2,7 & - & - \\
\hline Protomyctophum chilensis & 26,5 & 2,9 & - & - & - \\
\hline Stromateus stellatus & 21,6 & 2,9 & 10,8 & - & - \\
\hline Odontesthes regia & 17,4 & 5,9 & 5,4 & - & - \\
\hline
\end{tabular}

El mar interior limitado al oriente por la isla Chiloé está conectado al océano por dos aberturas directas, el canal Chacao y la boca del Guafo (Fig. 1). Por esta última conexión entra la onda mareal dos veces al día (marea semidiurna) que se propaga hacia el norte, y por efecto de Coriolis se mueve por el lado oriental de la isla Chiloé hasta el golfo de Ancud (Cáceres et al. 2003). A través de este mecanismo, las corrientes mareales generan fricción con el fondo, y por ser zonas más someras la turbulencia generada por la marea puede propagarse en toda la columna de agua, disminuyendo la estratificación. Además, eventos de viento fuerte pueden producir mezcla vertical turbulenta que aumenten la profundidad de la capa de mezcla (hasta $80 \mathrm{~m}$ de profundidad), disminuyan la concentración de clorofila (Delgado \& Marín 2006) y dispersen los parches de huevos y larvas de peces. Por otro lado, el efecto de la mezcla mareal es menos notorio en el borde occidental de la cuenca interior de Chiloé debido a su mayor profundidad y al mayor aporte de agua dulce de la cordillera, aunque el estrés del viento puede generar mezcla localmente. Sin embargo, los fuertes gradientes horizontales y verticales de salinidad (Silva et al. 1998) aumentan la estabilidad de la columna de agua, reducen la advección del plancton (generando retención larval) y limitan el efecto del viento a los primeros metros de la columna de agua (10$30 \mathrm{~m}$ de profundidad).

Las diferencias en las características topográficas y oceanográficas de ambos bordes de la cuenca pueden tener efectos en las respuestas biológicas de las especies marinas presentes en el área. La turbulencia a pequeña escala en ambientes estratificados puede afectar positivamente las tasas de encuentro predadorpresa y estado de condición de larvas y juveniles de peces, en comparación con zonas bien mezcladas (Lough \& Mountain 1996, Lee et al. 2005). La estabilidad de la columna de agua también puede afectar positivamente el crecimiento de los peces durante todo su ciclo de vida, y en consecuencia alterar la probabilidad de sobrevivencia limitando la 
susceptibilidad a la predación e inanición (Lee et al. 2006). Una relación significativamente positiva se ha encontrado entre larvas pequeñas $(<5 \mathrm{~mm})$ de merluza Merluccius bilinearis y el valor de $\mathrm{N}^{2}$, lo que explica la relación entre la distribución vertical de estas larvas y la naturaleza estratificada de la columna de agua (Reiss et al. 2002).

Los resultados obtenidos muestran que especies de peces de diferentes hábitats utilizan zonas de alta estratificación al interior de los fiordos como áreas de desove y crianza larval en el mar interior de Chiloé. Esta tendencia es similar a descripciones realizadas para otros sistemas de fiordos alrededor del mundo, donde diferentes especies de peces de origen demersal (lenguados Atheresthes stomias e Hippoglossus stenolepis, Bailey \& Picquelle 2002; merluza Merluccius productus, Fulmer \& Bollens 2005), pelágico (arenque Clupea harengus, Stenevik et al. 1996) y mesopelágico (pez hacha Maurolicus muelleri, Lopes 1979) completan sus ciclos de vida en zonas de fuerte estratificación vertical. Posteriores investigaciones deben tratar de definir si esta selección es realizada directamente por los adultos (selección de zonas de desove) o se produce indirectamente por mortalidades diferenciales de los estados tempranos.

Estudios previos han mostrado que el mar interior de Chile austral es una importante área de desove y crianza larval temprana de merluza de cola Macruronus magellanicus y de merluza del sur Merluccius australis comparable en abundancia a los focos de desove al sur de la boca del Guafo (Balbontín \& Bernal 1997, Bustos et al. 2007). Además, los resultados obtenidos en el presente estudio indican una abundancia de huevos de anchoveta y sardina comparables a las zonas de desove de Chile central (Cubillos et al. 2007), y mayor que las abundancias ictioplanctónicas obtenidas en aguas interiores de la XI región (Balbontín \& Bernal 2005, Córdova \& Balbontín 2006, Landaeta \& Castro 2006). Estas características hacen del mar interior de la Región de Los Lagos una importante área reproductiva para peces marinos a escala regional.

Finalmente, al comparar estos resultados con datos de ictioplancton de un crucero oceanográfico llevado a cabo en la misma área 10 años antes (Cimar-Fiordos 1), durante octubre de 1995, es posible notar el cambio en la composición taxonómica de las especies de larvas de peces. Durante octubre de 1995 dominaron las larvas de especies de origen mesopelágico (M. parvipinnis) y demersal (Macruronus magellanicus, Merluccius australis), alcanzando un 54 y $28 \%$ de la abundancia larval total, respectivamente, y que también fueron recolectadas principalmente en zonas de fiordos continentales con fuerte estratificación (Balbontín \& Bernal 1997, Bustos et al. 2007). A pesar de presentarse condiciones oceanográficas similares durante ambos cruceros (Silva et al. 1998, Fig. 5), las proporciones de estos grupos variaron considerablemente en noviembre de 2005, cuando las larvas de peces pelágicos pequeños alcanzaron el $65 \%$ del total de larvas, encontrándose además estados tempranos de sardina fueguina Sprattus fueguensis, especie de la cual recientemente se han detectado grandes cardúmenes en el mar interior de Chiloé $^{2}$. Para entender si estas diferencias obedecen a variabilidad de pequeña escala en las condiciones locales y reproductivas de las poblaciones locales de peces, o a diferencias de mayor escala (i.e., interdecadal) similares a las descritas en latitudes altas del Hemisferio Norte (Peterson \& Schwing 2003) o a cambios gatillados por la intensiva actividad de acuicultura en la zona (Buschmann et al. 2006), es necesario implementar un plan de monitoreo biooceanográfico permanente en la zona, dada su importancia para el manejo de varios recursos pesqueros relevantes en la zona sur de Chile.

\section{AGRADECIMIENTOS}

Este trabajo fue financiado por el proyecto C11F 05-02 del Comité Oceanográfico Nacional (CONA) adjudicado a FB. Durante la escritura del manuscrito la primera autora fue financiada por Programa FNDR del Gobierno Regional de Los Lagos, BIP: 30038191-0 y el Proyecto Regional CONICYT: R04I1003.

2 ARANÍS A, L CABALLERO, F CERNA, A GÓMEZ, A LÓPEZ \& C BERNAL (2006) Monitoreo de la pesquería de pequeños pelágicos en aguas interiores de la X Región, año 2004. Informe Final FIP 2004-39, 231 pp. Disponible en: www.fip.cl 


\section{LITERATURA CITADA}

ACHA EM, HW MIANZAN, RA GUERRERO, M FAVERO \& J BAVA (2004) Marine fronts at the continental shelves of austral South America: physical and ecological processes. Journal of Marine Systems 44: 83-105.

BAILEY KM \& SJ PICQUELLE (2002) Larval distribution and offshore spawning flatfish in the Gulf of Alaska: potential transport pathways and enhanced onshore transport during ENSO events. Marine Ecology Progress Series 236: 205-217.

BALBONTÍN F \& R BERNAL (1997) Distribución y abundancia del ictioplancton en la zona austral de Chile. Ciencia y Tecnología del Mar 20: 155-163.

BALBONTÍN F \& R BERNAL (2005) Cambios estacionales en la composición y abundancia del ictioplancton de los canales australes entre el Golfo Corcovado y Golfo Elefantes, Chile. Ciencia y Tecnología del Mar (Chile) 28: 99-111.

BALBONTÍN F \& R BRAVO (1993) Fecundidad, talla de primera madurez sexual y datos biométricos en la merluza del sur, Merluccius australis. Revista de Biología Marina (Chile) 28: 111-132.

BALBONTÍN F, F URIBE, R BERNAL \& M BRAUN (2004) Descriptions of larvae of Merluccius australis, Macruronus magellanicus, and observations on a larva of Micromesistius australis from southern Chile. New Zealand Journal of Marine and Freshwater Research 38: 609-619.

BUSCHMANN AH, VA RIQUELME, MC HERNÁNDEZGONZÁLEZ, D VARELA, JE JIMÉNEZ, LA HENRÍQUEZ, PA VERGARA, R GUÍNEEZ \& L FILÚN (2006) A review of the impacts of salmonid farming on marine coastal ecosystems in the southeast Pacific. ICES Journal of Marine Science 63: 1338-1345.

BUSTOS CA \& MF LANDAETA (2005) Desarrollo de huevos y larvas tempranas de la merluza del sur, Merluccius australis, cultivados bajo condiciones de laboratorio. Gayana (Chile) 69: 402-408.

BUSTOS CA, F BALBONTÍN \& MF LANDAETA (2007) Spawning of the southern hake Merluccius australis (Pisces: Merluccidae) in Chilean fiords. Fisheries Research 83: 23-32.

CÁCERES M, A VALLE-LEVINSON \& L ATKINSON (2003) Observations of cross-channel structure of flow in an energetic tidal channel. Journal of Geophysical Research 108: 1-10.

CIECHOMSKI J (1972) Estudios sobre los huevos y larvas de la sardina fueguina, Sprattus fueguensis y de Maurolicus muelleri, hallados en aguas adyacentes al sector patagónico argentino. Physis (Argentina) 30: $557-567$

CÓRDOVA G \& F BALBONTIN (2006) Distribución espacial de la abundancia y de la talla de ocho tipo de larvas de peces entre la Boca del Guafo y Bahía Anna Pink, zonas austral de Chile. Ciencia y Tecnología del Mar (Chile) 29: 153-161.

CUBILLOS LA, P RUIZ, G CLARAMUNT, S GACITÚA, S NÚNEEZ, LR CASTRO, K RIQUELME, C ALARCÓN, C OYARZÚN \& A SEPÚLVEDA (2007) Spawning, daily egg production, and spawning stock biomasa estimation for common sardine (Strangomera bentincki) and anchovy (Engraulis ringens) off central southern Chile in 2002. Fisheries Research 86: 228-240.

DELGADO LE \& VH MARÍN (2006) Determinación de zonas de alta concentración de clorofila-s en la región norte de los fiordos y canales australes (crucero CIMAR-9 Fiordos) por medio de sensoramiento remoto. Ciencia y Tecnología del Mar (Chile) 29: 87-94.

ESCHMEYER W (2007) The catalog of fishes on-line. California Academy of Sciences, USA. http:// www.calacademy.org/research/ichthyology/catalog/ . $(10 / 07 / 2007)$

FISCHER W (1958) Primeras fases del desarrollo del blanquillo (Prolatilus jugularis). Revista de Biología Marina (Chile) 8: 1-24.

FULMER JH \& SM BOLLENS (2005) Responses of the chaetognath Sagitta elegans, and larval Pacific hake, to spring diatom and copepod bloom in a temperate fjord (Dabob Bay Washington). Progress in Oceanography 67: 442-461.

HARRIS SA \& DP CYRUS (2000) Comparison of larval fish assemblages in three large estuarine systems, Kwazulu-Natal, South Africa. Marine Biology 137: 527-541.

HERRERA G, E TARIFEÑO \& MC ORELLANA (1987) Descripción de huevos y primeras fases larvales de la sardina común (Strangomera bentincki) y del machuelo (Ethmidium maculatum). Biología Pesquera (Chile) 16: 107-113

IRIARTE JL, HE GONZALEZ, KK LIU, C RIVAS \& C VALENZUELA (2007) Spatial and temporal variability of chlorophyll and primary productivity in surface waters of southern Chile $\left(41.5-43^{\circ} \mathrm{S}\right)$. Estuarine, Coastal and Shelf Science 74: 471-480.

JAGER Z (2001) Transport and retention of flounder larvae (Platichthys flesus L.) in the Dollard nursery (Ems estuary). Journal of Sea Research 45: 153171.

LANDAETA MF \& LR CASTRO (2002) Spring spawning and early nursery zone of the mesopelagic fish Maurolicus parvipinnis at the coastal upwelling zone off Talcahuano, central Chile. Marine Ecology Progress Series 226: 179-191.

LANDAETA MF \& LR CASTRO (2004) Zonas de concentración de ictioplancton en el archipiélago de Juan Fernández, Chile. Ciencia y Tecnología del Mar (Chile) 27: 43-53.

LANDAETA MF \& LR CASTRO (2006) Larval distribution and growth of the rockfish, Sebastes capensis (Sebastidae, Pisces), in the fjords of southern Chile. ICES Journal of Marine Science 63: 714-724.

LANDAETA MF, GA HERRERA, M PEDRAZA, CA BUSTOS \& LR CASTRO (2006) Reproductive tactics and larval development of bigeye flounder, Hippoglossina macrops off central Chile. Journal of Marine Biological Association of the United Kingdom 86: 1253-1264.

LANKSBURY JA, JT DUFFY-ANDERSON, KL MIER \& MT WILSON (2005) Ichthyoplankton abundance, distribution and assemblage structure in the Gulf of Alaska during September 2000 and 2001. Estuarine, Coastal and Shelf Science 64: 775-785

LEE O, RDM NASH \& BS DANILOWICZ (2005) Smallscale spatio-temporal variability in ichthyoplankton and zooplankton distribution in relation to a tidalmixing front in the Irish Sea. ICES Journal of Marine Science 62: 1021-1036.

LEE O, BS DANILOWICZ \& M DICKEY-COLLAS (2006) Temporal and spatial variability in growth and condition of dab (Limanda limanda) and sprat (Sprattus sprattus) larvae in the Irish Sea. Fisheries Oceanography 15(6): 490-507.

LOPES PC (1979) Eggs and larvae of Maurolicus muelleri (Gonostomatidae) and other fish eggs and larvae 
from two fjords in western Norway. Sarsia 64: 199210.

LOUGH RG \& DG MOUNTAIN (1996) Effect of smallscale turbulence on feeding rates of larval cod and haddock in stratified water on George Bank. Deep Sea Research II 43: 1745-1772.

MOSER HG (1996) The early stages of fishes in the California Current region. CalCOFI Atlas $\mathrm{N}^{\circ} 33$. Allen Press Inc., Lawrence, Kansas, USA. 1,505 pp.

NEIRA FJ, AG MISKIEWICZ \& T TRNSKI (1998) Larvae of temperate Australian fishes. Laboratory guide for larval fish identification. University of Western Australia Press, Nedlands, Australia. 474 pp.

ORELLANA MC \& F BALBONTÍN (1983) Estudio comparativo de las larvas de clupeiformes de la costa de Chile. Revista de Biología Marina (Chile) 19: $1-46$

PALMA S \& N SILVA (2004) Distribution of siphonophores, chaetognaths, euphausiids and oceanographic conditions in the fjords and channels of southern Chile. Deep Sea Research II 51: 513 535.

PETERSON WT \& FB SCHWING (2003) A new climate regime in northeast Pacific ecosystems. Geophysical Research Letters 30: 1-6.
REISS CS, A ANIS, CT TAGGART, JF DOWER \& B RUDDICK (2002) Relationship among vertically structured in situ measures of turbulence, larval fish abundance and feeding success and copepods on Western Bank, Scotian Shelf. Fisheries Oceanography 11: 156-174

SILVA N, C CALVETE \& H SIEVERS (1998) Masas de agua y circulación general para algunos canales australes entre Puerto Montt y Laguna San Rafael, Chile (Crucero Cimar-Fiordo 1). Ciencia y Tecnología del Mar (Chile) 20: 17-48.

STENEVIK EK, P FOSSUM, A JOHANNESSEN \& A FOLKVORD (1996) Identification of Norwegian spring spawning herring (Clupea harengus L.) larvae from spawning grounds off western Norway applying otolith microstructure analysis. Sarsia 80: 285-292.

URIBE F \& F BALBONTÍN (2005) First description of larvae of Bathylagichthys parini (Pisces, Bathylagidae) from the southeastern Pacific. Bulletin of Marine Science 77: 201-207.

YANNICELLI B, LR CASTRO, W SCHNEIDER \& M SOBARZO (2006) Crustacean larvae distribution in the coastal upwelling zone off central Chile. Marine Ecology Progress Series 319: 175-189. 
